\title{
Hérnia Incisional: proposta de um fluxograma que oriente o tratamento
}

\section{Incisional Hernia: proposal for a flow chart to guide treatment}

\author{
Aleksandra Markovic ${ }^{1}$, Márcio Alencar Barreira², Annya Costa Araújo de Macedo Goes ${ }^{3}$ \\ 1. Médica Estrangeira (Universidade de Belgrado, Sérvia) pós-graduando da residência cirurgia geral do Hospital Walter Cantídio da Universidade Federal \\ do Ceará. Brasil. 2. Cirurgião e Discente do Programa de Mestrado Profissional em Tecnologia Minimamente Invasiva e Simulação pelo Centro Universitário \\ Christus (UNICHRISTUS), Brasil. 3. Cirurgiã do Hospital Walter Cantídio e Supervisora do Programa de Pós-graduação em Cirurgia Geral e Coordenadora da
} Residência Médica da Universidade Federal do Ceará (UFC), Brasil.

\section{Resumo}

Introdução: A hérnia incisional (HI) é conceituada como qualquer falha ou orifício na parede abdominal, com ou sem protuberância, em área de cicatriz pós-operatória, diagnosticada pelo exame clínico ou de imagem. Objetivo: criar um fluxograma que, embasado por uma relevante bibliografia, oriente o tratamento de tais hérnias. Método: Trata-se de um estudo de caráter descritivo, exploratório e observacional retrospectivo, tipo revisão integrativa. 0 período de coleta de dados desenvolveu-se entre os meses de janeiro e setembro de 2016, nas seguintes bases de dados: Biblioteca Virtual da Saúde: Scielo (Scientific Eletronic Library Online) e PubMed (Medical Literature Analysis and Retrieval System Online) - free full text e em língua portuguesa e inglesa - com publicações realizadas no período entre 2000 e 2015, por meio do seguinte descritor: incisional hernia review. Como critério de inclusão, foram selecionados os artigos cujo título indicasse alguma forma de diagnóstico, tratamento ou abordagem de HI. Após a leitura do Abstract, foram excluídos das propedêuticas os artigos que, em sua metodologia, não se apresentassem como revisão sistemática ou metanálise, culminando em nove artigos. Para a coleta de informação, todos os artigos foram lidos em sua íntegra, visando às mais variadas abordagens de HI em várias situações e sempre conferindo a referência bibliográfica. Assim, foram avaliados os artigos citados como referência entre os nove selecionados, mesmo que fossem de anos inferiores a 2000. Resultados: Foram localizados 42 artigos no Scielo e 351 no Pubmed. Foram obtidos nove artigos entre os anos 2000 e 2015 e 21 de anos anteriores. O diagnóstico, na maioria dos casos, foi clínico. Geralmente, o tratamento é cirúrgico, podendo ser por via aberta ou laparoscópica. Em ambos os casos, o uso de prótese é quase mandatório, salvo algumas exceções. Quanto maior o tamanho da hérnia, mais se tende indicar a via aberta. Nos tamanhos maiores que $15 \mathrm{~cm}$, o estudo da pressão abdominal é necessário, também nos estudos com pressão maior que $10 \mathrm{cmH} 20$ que sejam indicativos de procedimentos prévios ao reparo definitivo da hérnia, tais como o pneumoperitônio progressivo e a aplicação intraparietal de toxina botulínica tipo A. Nos casos de urgência, como o estrangulamento e a síndrome compartimental, a laparotomia imediata se faz necessária. Conclusões: Por meio deste estudo, pudemos criar um verdadeiro roteiro para o tratamento das Hérnias Incisionais. Tal fluxograma poderia ser adotado em qualquer centro hospitalar que cuida de tal patologia, uma vez que ele segue as recomendações vigentes em vários artigos científicos especializados no tema.

Palavras-chave: Hérnia Incisional. Fluxograma. Tratamento.

\section{Abstract}

Introduction: Incisional hernia is conceptualized as any defect or orifice in the abdominal wall, with or without protuberance, in post-operative scar area, diagnosed in clinical examination or imaging techniques. Objective: create a flowchart which, based on the relevant bibliography, can be used as a guideline for the treatment of such hernias; Methods: This is a retrospective study of descriptive, exploratory and observational nature, in the manner of integrative review. The period of data collection was from January to September 2016 in the following databases: Virtual Health Library: Scielo (Scientific Electronic Library Online) and PubMed (Medical Literature Analysis and Retrieval System On-line) - free full text, in English and in Portuguese - regarding publications going from 2000 to 2015 with the following descriptor: incisional hernia review. The criterion of inclusion was the selection of articles whose titles indicated some form of IH diagnosis, treatment or approach. After reading the Abstract of the articles, we excluded those whose methodology was not a systematic review or meta-analysis, culminating in 9 articles. All articles were read in full, focusing on the different IH approaches in several situations and, in each case, examining the bibliographic reference. Thus, we examined articles indicated as reference in the 9 selected articles even when they were prior to 2000. Results 42 articles were found in Scielo and 351 in PubMed. The result was 9 articles between 2000 and 2015 and 21 articles related to previous years. In most cases, the diagnosis is clinical. The treatment is usually surgical, either in open surgery or laparoscopy. In both cases, the use of prosthetic meshes is practically mandatory, with some exceptions. The larger the hernia is, the more open surgery is recommended. In sizes above $15 \mathrm{~cm}$ ( 5.91 inches) it is necessary to examine the abdominal pressure and if it is higher than $10 \mathrm{cmH} 20$ other procedures before the definitive hernia repair are recommended, such as progressive pneumoperitoneum and intraparietal application of botulinum toxin type A. In emergency cases, such as strangulation and compartmental syndrome, immediate laparotomy is required. Conclusions: This study enabled the creation of a guideline for the treatment of Incisional Hernias. The resulting flowchart could be adopted in any hospital center that addresses this pathology, since it follows the current recommendations of several specialized scientific articles.

Keywords: Incisional Hernia. Flowchart. Treatment.

INTRODUÇÃO

A hérnia incisional ( $\mathrm{HI}$ ) é definida como qualquer falha ou orifício na parede abdominal, com ou sem protuberância, em área de cicatriz pós-operatória, diagnosticada pelo exame clínico ou de imagem. É a única hérnia da parede abdominal que pode ser considerada iatrogênica por natureza, sendo a maioria diagnosticada no primeiro ano de evolução pós-operatória. Entretanto, na literatura, há relatos do aparecimento de HI após 5 anos de um procedimento cirúrgico ${ }^{1}$. 
Sua incidência e frequência de aparecimento dependem de vários fatores, ocorrendo, sobretudo, em pacientes do sexo feminino, obesos, após cirurgia do aparelho digestivo, ginecológica, obstétrica e de urgência².

Nos Estados Unidos da América, cerca de 400.000 pacientes são operados por Hérnia Incisional por ano. O reparo cirúrgico com auxílio de tela sintética pode reduzir significativamente o risco de recidiva. No entanto, mesmo com o reparo com tela, a taxa de recorrência em 10 anos chega a 32\%, e os índices de reoperação vêm sendo relatados em cerca de $23 \%^{3}$.

A incidência da $\mathrm{HI}$ varia de acordo com as incisões realizadas anteriormente. A localização mais frequente é após as incisões medianas, sobretudo, após laparotomia de urgência. Em seguida, vêm as HI pós-incisões transversa/oblíquas, utilizadas em procedimentos como cirurgias do trato biliar ${ }^{2}$ (Tabela 1).

Tabela 1. Taxas de hérnia incisional após diferentes tipos de incisão

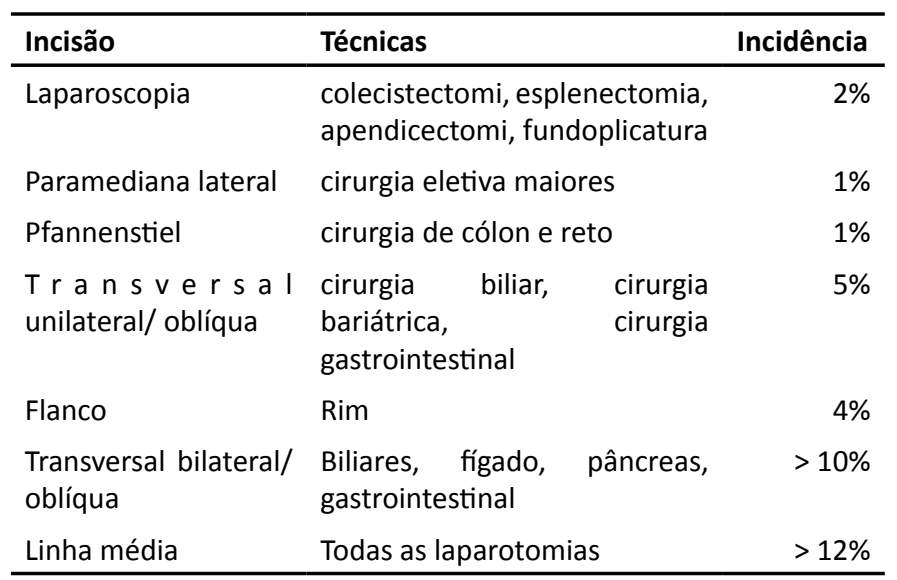

Fonte: Khaiara HS, Lall P, Hunter B, Brown JH. Repair of incisional hernias. J R Coll Surg Edinb. 2001;46:39-43. Chapter 7. Pag 84

Os fatores mais frequentemente responsáveis por uma hérnia incisional são: técnica cirúrgica inadequada, infecção de ferida operatória, idade avançada, desnutrição, obesidade, tabagismo, uso de corticosteroides, medicações antineoplásicas, sepse e presença de patologias crônicas ${ }^{2}$.

A idade avançada parece agir como um fator de risco independente, apesar de sua análise ser dificultada pela coexistência de doenças crônicas nos pacientes idosos, como: DPOC, insuficiência renal e diabetes mellitus descompensado (que altera o processo normal de cicatrização) ${ }^{4}$.

Como sintomatologia, é frequente a presença de tumoração abrangendo parte ou toda a cicatriz cirúrgica, podendo até ultrapassar o local da cicatriz em todo o seu contorno, chegando a imensas dimensões ${ }^{1,5}$. Distensão e atonia das vísceras ocas podem ocorrer e causar dificuldade de micção e evacuação ${ }^{6}$.

Com o passar do tempo, a tendência habitual das hérnias incisionais é aumentar, progressivamente, levando a um aumento de complicações (encarceramento, estrangulamento, alterações cutâneas) e prejuízo da qualidade de vida com limitação da prática de exercícios físicos ${ }^{7}$.

O diagnóstico é clínico por meio de uma anamnese direcionada e exame fisíco do paciente em decúbito dorsal e em pé (Figura 1). Eventualmente, há necessidade de complementação com exames de imagens, em especial, em pacientes obesos, sendo os mais utilizados a ultrassonografia e a tomografia computadorizada.

Figura 1. Paciente com hérnia incisional

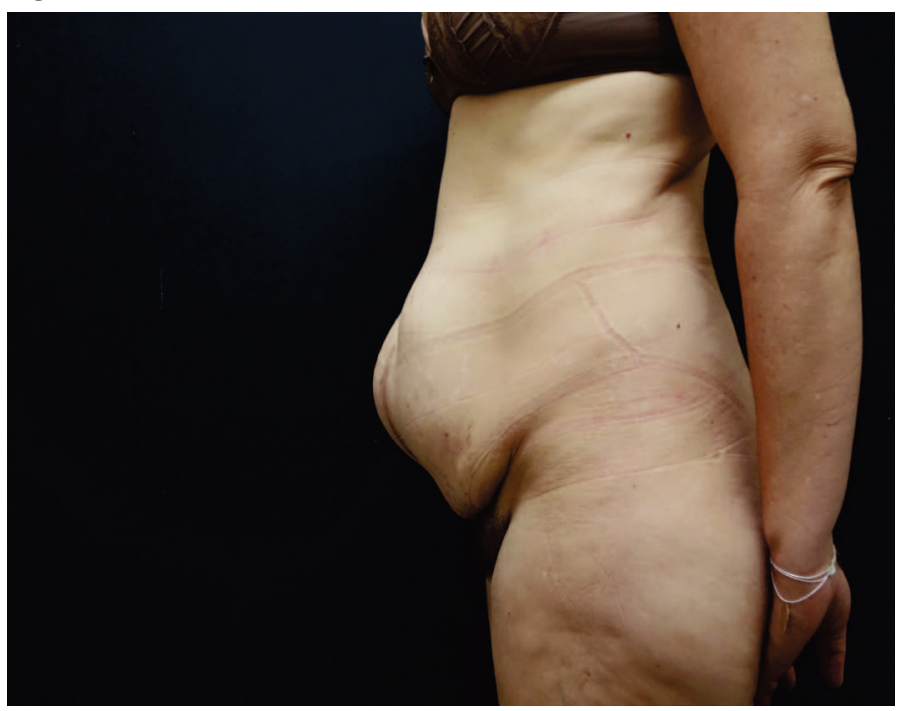

Fonte: Arquivo do Autor. Foto autorizada pelo paciente.

No estudo realizado por Gutierrez de la Pena, Vargas e Dieguez, em $2001^{8}$, o diagnóstico correto foi dado em $88 \%$ no exame físico e $98 \%$ com auxílio da tomografia. O estudo realizado por Beck et al., em $2013^{9}$, que avaliou a eficácia comparativa da ultrassonografia abdominal dinâmica em relação à tomografia para o diagnóstico das hérnias, demonstrou uma sensibilidade de $98 \%$ e especificidade de $88 \%$ para a ultrassonografia dinâmica, que identificou mais hérnias que a tomografia. Todavia, a tomografia apresentou maior especificidade, de $97 \%$, com sensibilidade de $91 \%$.

A alta incidência de recidiva, a morbidade relacionada ao tratamento dos pacientes com hérnia incisional e a importância de uma conduta padronizada para o tratamento dos pacientes com essa afecção cirúrgica mostram que existe necessidade de se normatizar o tratamento desta patologia pela criação de uma representação esquemática de fácil compreensão.

O objetivo principal deste trabalho foi desenvolver um fluxograma que oriente o tratamento dos pacientes com hérnia incisional, além de discutir pontos fundamentais no tratamento e contribuir para a padronização das condutas.

\section{MATERIAL E MÉTODOS}

Trata-se de um estudo de caráter descritivo, exploratório e observacional retrospectivo de recuperação e análise crítica da literatura, pautado em um revisão literária, tipo revisão 
integrativa.

O período de coleta de dados desenvolveu-se durante os meses entre janeiro de 2016 e setembro de 2016, nas seguintes bases de dados: Biblioteca Virtual da Saúde: Scielo (Scientific Eletronic Library Online) e PubMed (Medical Literature Analysis and Retrieval System Online) - free full text em língua portuguesa e inglesa, com publicações realizadas no período entre 2000 e 2016, por meio do seguinte descritor: incisional hernia review. Foram localizados 42 artigos no Scielo e 351 no Pubmed. Como critério de inclusão, foram selecionados os artigos no qual o título indicasse alguma forma de diagnóstico, tratamento ou abordagem de HI. Após a leitura do Abstract, foram excluídos de nossa propedêutica os artigos que, em sua metodologia, não se apresentassem como revisão sistemática ou metanálise, culminando em nove artigos. Para a coleta de informação, todos os artigos foram lidos em sua íntegra, visando às mais variadas abordagens de $\mathrm{HI}$ em várias situações e sempre conferindo a referência bibliográfica. Assim, foram avaliados os artigos citados como referência entre os nove selecionados, mesmo que fossem de anos inferiores a 2000. Foram obtidos nove artigos entre os anos 2000 e 2015 e 21, de anos anteriores.

Para a análise das informações, foi realizada a organização do conteúdo encontrado quanto ao ano, tipo de publicação e abordagem metodológica, essência do conteúdo/produção do conhecimento e recomendações dos autores.

Com base nesses dados, foi confeccionado, ao final do artigo, um fluxograma de abordagem das hérnias incisionais, que poderá servir de protocolo para qualquer hospital que tenha interesse em abordar tal patologia.

Salientamos que houve autorização por escrito dos pacientes para terem suas fotos expostas neste trabalho.

\section{RESULTADOS E DISCUSSÃO}

A hérnia incisional deve ser tratada precocemente e de forma cirúrgica. Apenas os pacientes de risco cirúrgico elevado ou que não desejem ser submetidos ao tratamento cirúrgico podem ter seus sintomas controlados por uma cinta ${ }^{1,4}$.

Antes de indicar um procedimento, é prudente classificar a HI. Todavia, às vezes, isso torna-se difícil em razão de sua grande diversidade e heterogenicidade ${ }^{5,6}$. Uma classificação simples cujos dados são prontamente obtidos é a de Chevrel e Rath ${ }^{10}$, que propuseram três parâmetros para classificar as HI: localização (mediana e lateral), tamanho (considerando que a largura do defeito é mais importante que a superfície, o comprimento ou tamanho do saco herniário) e o número de recidivas. A hérnia com largura inferior a $3 \mathrm{~cm}$ é considerada pequena, entre 3 e $10 \mathrm{~cm}$ média, de 10 a $15 \mathrm{~cm}$ grande e acima desse valor gigante ${ }^{1}$.

Silva ${ }^{11}$ prefere classificar as hérnias por meio de características clínicas (Tabela 2).
A classificação European Hernia Society (EHS), proposta no 29응 Congresso da Sociedade Europeia de Hérnia em Atenas, utiliza, sobretudo, parâmetros anatômicos, sendo, primeiramente, questionado se a $\mathrm{HI}$ é em linha mediana (subxifoidea, epigástrica, umbilical, infraumbilical e suprapúbica) ou se é lateral (subcostal, flanco, ilíaca e lombar). Em seguida, avalia-se se há recorrência e o tamanho da HI (largura e comprimento) ${ }^{12}$.

Tabela 2. Classificação de Hérnia Incisional.

\begin{tabular}{|c|c|}
\hline ESPONTÂNEAS & \\
\hline Raquítica & $\begin{array}{l}\text { Linha alba distendida e } \\
\text { proeminente }\end{array}$ \\
\hline Paralítica & $\begin{array}{l}\text { Devido à lesão radiculo } \\
\text { medular ou de nervos } \\
\text { periféricos }\end{array}$ \\
\hline Diastática & $\begin{array}{l}\text { Devido à diástase dos } \\
\text { músculos retos do abdome }\end{array}$ \\
\hline TRAUMÁTICA & \\
\hline Acidental & $\begin{array}{l}\text { Trauma direto, ruptura } \\
\text { da parede ou perda de } \\
\text { substância }\end{array}$ \\
\hline Pos-operatória & $\begin{array}{l}\text { Causa mais frequente de } \\
\text { Hérnia Incisional }\end{array}$ \\
\hline CONGÊNITA & \\
\hline $\begin{array}{l}\text { Ex: Síndrome de "prune } \\
\text { belly"(deficiência } \\
\text { musculatura da } \\
\text { abdominal) }\end{array}$ & \\
\hline
\end{tabular}

Fonte: Colégio brasileiro de cirurgiões, Hérnias da Parede Abdominal, Alcino Lázaro da Silva, Ano III-Volume I,1997, capítulo 8, pagina 83

\section{PREPARO PRÉ-OPERATÓRIO}

O preparo mecânico do cólon é recomendado em poucas situações, tais como presença de múltiplas aderências ou se o conteúdo herniário tiver alças intestinais ${ }^{13}$. A cateterização nasogástrica é recomendada, sobretudo nas cirurgias laparoscópicas, para se evitar acidentes de punção com os trocáteres ${ }^{13}$.

Quanto à antibioticoterapia pré-operatória para os pacientes que utilizarão tela, uma única dose de Cefalosporina de primeira geração (Cefazolina) deve ser dada na indução anestésica. Devese adicionar Vancomicina em pacientes colonizados por MRSA. A Vancomicina ou Clindamicina deve ser dada a pacientes alérgicos às Cefalosporinas ${ }^{14}$.

Nos casos das hérnias grandes, devemos realizar um estudo da pressão intra-abdominal (PIA) com a realização da redução externa das alças intestinais e utilização de uma cinta abdominal. A aferição da PIA é realizada por uma sonda vesical que é conectada a um sistema de PVC (Pressão Venosa Central) ${ }^{15}$.

Nos casos de hérnias incisionais com graus elevados de pressão, 
podemos optar por uma entre as seguintes estratégias: o pneumoperitônio progressivo (PPP) e a aplicação intraparietal de toxina botulínica tipo A pré-operatória.

O PPP é um procedimento pré-operatório idealizado há mais de 60 anos para aumentar o volume da cavidade abdominal, de modo a possibilitar a reintrodução das vísceras ocas herniadas pela diminuição do edema mesentérico crônico, redução do volume das vísceras ocas e, com isso, realizar o fechamento sem tensão da parede abdominal. Esses cuidados permitem uma melhora progressiva da função do diafragma e promovem a lise de aderências intra-abdominais, ainda, no pré-operatório. Para tanto, são injetados um total de 15 a 20 litros de ar ou gás (CO2) em período de 3 a 6 semanas, período no qual, em geral, o paciente permanece internado ${ }^{15-18}$.

No caso da toxina botulínica, é realizada a infiltração de 50 UI da toxina do tipo A em cada lado da parede abdominal em torno de 4 semanas, antes do procedimento cirúrgico ${ }^{15-18}$.

A perda de peso pode ajudar a melhorar a situação técnica da operação e, consequentemente, reduzir a taxa de recorrência ${ }^{19}$. No entanto, não altera o risco de complicações perioperatórias ${ }^{19,20}$. É fundamnetal prevenir a trombose venosa profunda dos membros inferiores pelas medidas mecânicas e farmacológicas e pela deambulação precoce ${ }^{21}$. Após tais cuidados, decide-se quando e como operar.

\section{ABERTA x LAPAROSCÓPICA}

A correção laparoscópica de hérnia incisional, em comparação com o reparo aberto, tem uma menor taxa de infecções de feridas. Em médio/longo prazos de acompanhamento, as taxas de recorrência e de dor pós-operatória são semelhantes entre as duas técnicas, todavia, em curto prazo, tais taxas são menores nos procedimentos laparoscópicos ${ }^{22}$.

Em média, demonstrou-se um retorno ao trabalho em torno de 5 dias mais rápido após reparo laparoscópico ${ }^{22}$. Antes da escolha da técnica, o cirurgião deve lembrar-se dos recursos disponíveis em suas instituições e de sua experiência e formação com esta operação. Reparos anteriores, grandes defeitos e hérnia encarcerada aumentam a dificuldade e a duração do procedimento.

Conforme Cuccurullo et $a .^{23}$, são recomendadas cautelas nos defeitos maiores do que $10 \mathrm{~cm}$, mas não se consideram esses defeitos como contraindicação absoluta para o reparo laparoscópico ${ }^{23}$. Cuccurullo et al. recomendam que as hérnias com um tamanho menor que 3 centímetros não devem ser abordadas por laparoscopia ${ }^{23}$. Esta recomendação foi baseada em um levantamento mostrando que menos de $10 \%$ dos cirurgiões utilizam próteses (telas) em defeitos inferiores a 3 cm: "uma indicação indireta de um limite de tamanho mínimo para a laparoscopia"23.

O reparo laparoscópico leva a menos infecções cirúrgicas em relação ao reparo aberto e deve, portanto, ser considerado em pacientes com maior risco de infecção 22 . Independentemente se o reparo for aberto ou laparoscópico, o uso de dispositivos steri drape não diminui o índice de infecção pós-operatória 14. Situações especiais podem representar contraindicações para o reparo laparoscópico, tais como: presença de enxertos de pele abdominal, fístulas enterocutâneas ativas, necessidade de remover a prótese previamente colocada, grandes defeitos da parede abdominal, pequeno defeito, mas grande saco herniário e hérnia encarcerada/estrangulada ${ }^{23}$.

A correção cirúrgica da hérnia paraestomal é preferível por técnica aberta, pois tem uma alta taxa de recorrência. A melhor oportunidade para o reparo da hérnia é após o fechamento do estoma. Todavia, em casos em que não é possível o fechamento primário, Raymond e Abulafi (2002) recomendam a utilização de tela cortada especificamente para cada defeito, conseguindo, assim, entre 18 e 24 meses livres de recidiva ${ }^{24}$.

No mesmo tempo do fechamento da estomia, o reparo herniário não se relaciona com maiores taxas de recorrência e infecção local, porém, o manuseio de uma tela de material sintético em uma área de pele contaminada pelos efluentes do estoma parece temerário. Quando esse tipo de procedimento resulta em uma infecção, na maioria das vezes, ela evolui com difícil controle clínico e, frequentemente, requer a retirada da tela ${ }^{24}$.

\section{TÉCNICA OPERATÓRIA: LAPAROSCÓPICA}

A colocação do portal primário é idealmente no quadrante superior esquerdo ou direito, mas a localização deve ser modificada, de acordo com a história cirúrgica do paciente e anatomia. Os portais secundários devem ser feitos sob visão direta e o mais lateral possível à hérnia. A lise de aderências deve ser realizada com cuidado, evitando-se o uso inadvertido de eletrocautério. Os cirurgiões devem medir e documentar o tamanho do defeito herniário ${ }^{25}$.

É recomendado utilizar tela dupla face: como ComposixTM BardTM $^{\circledR}$ [composta de uma camada de politetrafluoretileno expandido (ePTFE), colocada em contato com as vísceras abdominais, e outra camada de polipropileno para contato com a parede abdominal] e ParietexTM Composite ${ }^{\circledR}$ (composta de uma camada de colágeno absorvível e outra não absorvível de poliéster) ${ }^{23}$.

Um tamanho da tela de, no mínimo, 3 a $4 \mathrm{~cm}$ maior que o da hérnia é necessário para permitir sobreposição da tela em relação ao defeito da parede abdominal. A fixação da prótese pode ser feita com suturas transfasciais e grampos ou apenas grampo. O tratamento do saco peritoneal e a colocação de drenos não são obrigatórios ${ }^{26}$.

\section{TÉCNICA OPERATÓRIA: ABERTA}

A correção primária com a utilização dos próprios tecidos somente é admitida nas hérnias pequenas (menores de 3 
$\mathrm{cm})$. Já as hérnias maiores necessitam do uso de prótese para correção cirúrgica ${ }^{5}$.

As principais opções com relação à localização da prótese são: pré-musculoaponeurótica (onlay), retromusculoaponeurótica (underlay ou sublay), nos bordos do defeito (inlay) e intraperitoneal ${ }^{5}$.

Em relação à técnica cirúrgica, suturas muito próximas das bordas da ferida, muito distantes uma das outras e sob muita tensão, são fatores que aumentam a probabilidade de deiscência. Algumas recomendações são importantes na profilaxia dessa complicação: devem-se utilizar fios monofilamentares e de material não absorvível (como o polipropileno), e a sutura deve ser contínua ou com pontos separados, desde que os pontos estejam próximos ( 1 a 1,5 cm de distância) e ancorados em boa quantidade de tecido aponeurótico (distância entre a borda da aponeurose e o fio de, aproximadamente, $2 \mathrm{~cm})^{4}$.

\section{Técnica Onlay}

Esta técnica consiste no posicionamento da tela sobre a aponeurose anterior do abdome, logo abaixo do subcutâneo na região pré-fascial ${ }^{1,4}$.

Como as outras técnicas, pode ser acompanhada da realização de avanço miofascial da parede abdominal, permitindo, desta forma, a correção desde hérnias simples até hérnias gigantes ${ }^{4}$.

Um aspecto muito importante desta técnica é a sobreposição de tela além do defeito ou overlap com, pelo menos, $5 \mathrm{~cm}$ em cada margem do defeito, bem como a fixação da tela com múltiplas suturas de fio absorvível, de preferência Poliglatina ${ }^{4,26}$. Com isso, há uma maior área de contato entre a tela e a parede, que facilita sua integração completa, diminuindo o vetor resultante de força sobre todos os pontos da parede ${ }^{26}$.

Com o uso de prótese de polipropileno, a recorrência na técnica Onlay varia de $3,4 \%$ a $24 \%{ }^{27}$. A taxa de infecção vai de $5 \%$ a $16 \%$, e a de recorrência de $2 \%$ a $11 \%{ }^{27}$.

\section{Técnica Inlay}

O posicionamento da tela nas bordas do defeito ou da parede (inlay) vem sendo progressivamente abandonado em função da alta incidência de recorrência e da menor taxa de integração do material sintético da malha ou tela com esta técnica, além de menor índice de satisfação com essa técnica, em função da diástase dos músculos retos do abdome ${ }^{28}$.

Os índices de recidiva podem chegar a $44 \%$ em correções de hérnias da linha média com o emprego na posição inlay. Fatores como a fixação da tela nas bordas do defeito, a ausência de sobreposição, a manutenção de diástase dos músculos retos e a manutenção do vetor de forças resultantes sobre a área do defeito herniário são fatores que acabaram levando este tipo de reparo a apresentar os piores resultados, o que faz que essa técnica esteja praticamente em desuso ${ }^{28}$.

\section{Técnica Sublay ou Retromuscular}

Originalmente, é denominada Técnica de Rives-Stoppa 29. Consiste na abertura da linha média, dissecção do saco herniário, abertura deste saco, lise de aderências entre o conteúdo abdominal e o saco herniário. A síntese ocorre com a abertura da bainha do músculo reto do abdome bilateralmente em sua face posterior, com dissecção ampla entre o músculo e sua bainha posterior até o seu final. As bainhas posteriores são aproximadas com sutura, sendo colocada uma tela sobre elas, posteriormente, aos músculos retos. Dá-se preferência às telas de polipropileno (Marlex ${ }^{\circledR}$ ou Prolene ${ }^{\circledR}$ ), que são preparadas individualmente e fixadas com fios não absorvível, tipo Prolene ${ }^{\circledR 28,29}$.

Helgstrand et al., em estudo observacional prospectivo realizado na Dinamarca, demonstraram menor taxa de recidiva em reparos sublay em relação aos onlay ${ }^{29}$.

\section{Técnica Intraperitoneal}

Teoricamente, a prótese intraperitoneal exibiria a maior resistência à pressão abdominal, pois a pressão forçaria a prótese de encontro à parede. O principal problema das próteses nessa localização deve-se ao risco potencial de lesões viscerais, como aderência de alças, obstrução e fistulização. Para reduzir estas complicações, preconiza-se a utilização de próteses compostas, sendo a face que fica em contato com o peritônio parietal macroporosa (polipropileno ou poliéster) e a outra em contato com a cavidade peritoneal de baixa porosidade para prevenção de aderências ${ }^{5,26}$.

A colocação da prótese intraperitonealmente na operação aberta tem como indicações as grandes hérnias incisionais, as multirrecidivadas, quando existirem lesões intra-abdominais associadas e pacientes obesos. Teria como vantagens tornar desnecessária a dissecação extensa da tela subcutânea e do tecido musculoaponeurótico. Apesar da alardeada proteção das próteses compostas contra aderências intestinais, recomendase, sempre que possível, interpor o omento maior entre as alças e a prótese ${ }^{5}$.

\section{MANEJO PÓS-OPERATÓRIO}

A dor deve ser tratada de forma escalonada com analgésicos, anti-inflamatórios, corticosteroides, injeção em ponto-gatilho ou bloqueio de nervo ${ }^{4}$.

O dreno abdominal é utilizado, sobretudo, nas cirurgias abertas quando se opta por uma extensa dissecção, permanecendo enquanto a drenagem estiver elevada. A retirada costuma ocorrer nos 4ㅇ ou 5 dias de pós-operatório quando a drenagem se reduz a menos de $30 \mathrm{ml}$ por $\mathrm{dia}^{4}$.

Os doentes que desenvolvem um íleo pós-operatório devem ser, inicialmente, tratados de modo conservador com a administração de líquidos, repouso intestinal e/ou 
descompressão gástrica ${ }^{26}$.

A heparinização é recomendada a pacientes que estão restritos ao leito, geralmente, por outras comorbidades, sendo as medidas mecânicas também utilizadas na profilaxia da trombose venosa, tais como meias elásticas ${ }^{4}$.

De preferência, o paciente recebe alta, utilizando uma cinta abdominal elástica, com utilização da cinta por cerca de três meses, tempo suficiente para a recuperacão da musculatura da parede abdominal 6 . O retorno às atividades cotidianas, como trabalhar, dirigir, ter relações sexuais, em média, ocorre no prazo de 10 dias, e a maioria dos pacientes volta às suas atividades normais em torno de três a seis meses, sendo o retorno às atividades mais precocemente com a cirurgia laparoscópica ${ }^{26}$.

\section{COMPLICAÇÕES}

Entre as complicações, a mais grave é a infecção da prótese que ocorre, particularmente, em pacientes com lesões cutâneas prévias (úlceras cutâneas, reação por corpo estranho devida a fios ou próteses de operações anteriores) e que pode levar à sua extrusão 1 (Figura 2). Antes de se planejar uma nova intervenção com a utilização de prótese, os processos infecciosos devem ser tratados $^{1,4}$.

Figura 2. Extrusão de Tela após infecção.

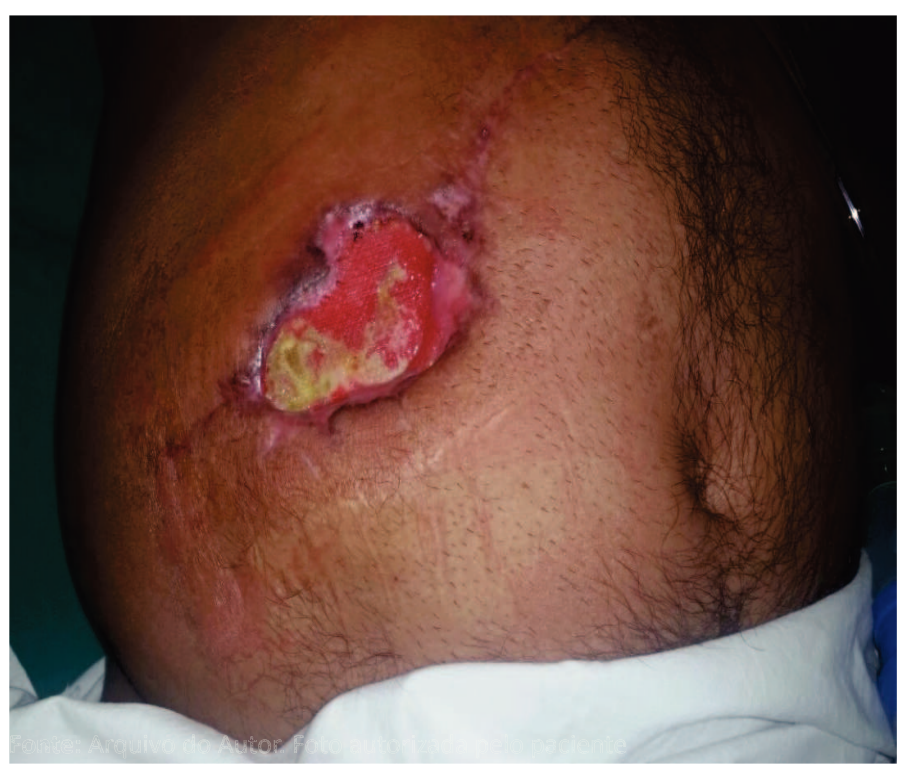

No caso de celulite pós-operatória, pode-se realizar um curso curto de antibióticos. Já no caso de infecção da prótese, pode ser tentada uma combinação de antibióticos, drenagem percutânea, desbridamento e terapia local com pressão negativa. Quando esta abordagem falha (ou em pacientes sépticos), a retirada da prótese deve ser realizada ${ }^{29}$.

O seroma é uma complicação esperada, e a maioria resolvese espontaneamente, sendo necessária a drenagem nos que persistirem ${ }^{4}$.

Nas hérnias incisionais grandes ou de longa duração, as vísceras perdem seus domínios no abdome. Neste caso, a recolocação das vísceras na cirurgia pode provocar compressão da veia cava inferior e insuficiência respiratória, por elevação forçada do diafragma ${ }^{30}$.

A taxa de recorrência pode chegar a 52\%, particularmente, com os procedimentos abertos ou aqueles em que se usam grampos em vez de suturas para o fechamento da ferida. Todavia, a taxa média de recidiva gira em torno de $10 \%$. Em um estudo realizado por Berg et al., 150 pacientes foram operados laparoscopicamente, encontrando quatro recorrências, três deles em razão de erros técnicos. Dois pacientes desenvolveram perfuração intestinal secundária após a lesão térmica, e nenhum apresentou infecção de ferida operatória29,30.

\section{CONSIDERAÇÕES FINAIS}

A indicação da correção das hérnias incisionais nos casos assintomáticos é controversa. Todavia, a tendência habitual de tais hérnias é aumentar progressivamente ao longo do tempo, o que eleva também ao índice de complicações e dificuldades técnicas em sua correção.

A correção precoce, sobretudo, quando tais hérnias ainda são de pequeno tamanho, além de apresentarem melhores resultados funcionais, podem dispensar a utilização de próteses ou serem realizadas por via laparoscópica sem a presença de deformidades cutâneas, como as observadas nas grandes hérnias. Todavia, apesar das modernas técnicas, há um número considerável de recidivas, muitas vezes, motivadas por complicações decorrentes do próprio ato cirúrgico.

Mesmo as correções bem-sucedidas não são isentas de inconvenientes. Por isso, é importante que o paciente seja alertado da possibilidade de sua expectativa em relação ao resultado, tanto estético como funcional, não ser alcançada.

Ressaltamos a importância dos cuidados técnicos no fechamento das incisões laparotômicas, na prevenção das infecções da ferida operatória, nos cuidados quanto às condições nutricionais e nas medidas que visam a controlar as afecções determinantes do aumento da pressão intra-abdominal na profilaxia das hérnias. Ressaltamos ainda a necessidade de um pré-operatório bemfeito, reduzindo os fatores de risco para a recidiva, tais como tabagismo e obesidade.

Após tudo o que foi exposto nesta revisão, confeccionamos um fluxograma que resume esquematicamente a conduta referente às hérnias incisionais (Figura 3).

A despeito do fluxograma, podemos ressaltar que o diagnóstico é clínico, ficando a ultrassonografia e a tomografia nos casos de dúvida no diagnóstico ou para estudo detalhado pré-operatório.

No caso de o paciente apresentar-se com evisceração, encarceramento ou estrangulamento no momento do diagnóstico de $\mathrm{HI}$, passa-se, de imediato, para a laparotomia como tratamento, em que o cirurgião deve estar preparado para 
enfrentar diversas situações, como uma necrose intestinal que necessite de enterectomia. De preferência, fecha-se a parede abdominal com auxílio de tela por técnica inlay.

No caso de o paciente não se apresentar como nas três situações acima, indica-se o tratamento cirúrgico eletivo, ficando o tratamento conservador (cinta elástica) para os pacientes que se recusarem a realizar a cirurgia ou não tiverem condições clínicas.

Figura 3. Fluxograma esquemático de conduta na HI.

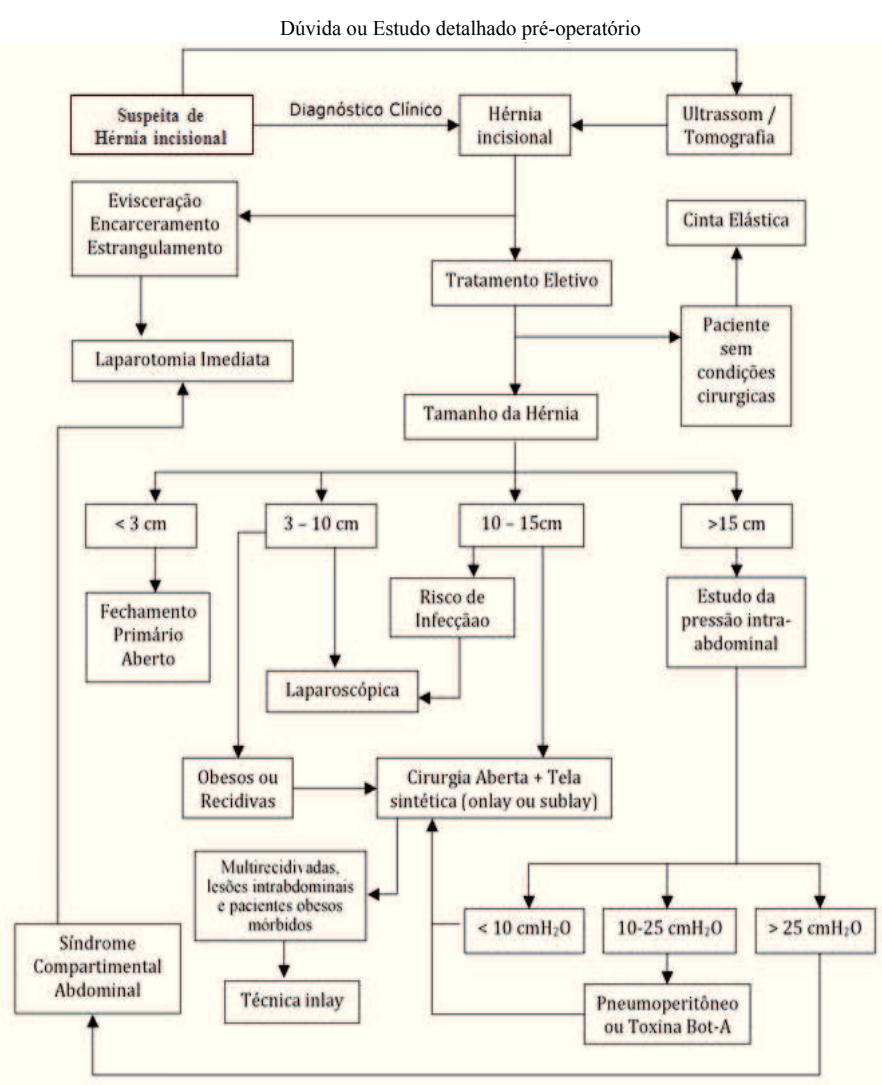

Fonte: Arquivo do Autor

De acordo com o tamanho da hérnia, orientamos o fechamento primário em $\mathrm{HI}$ menores que $3 \mathrm{~cm}$, utilizando telas sintéticas em defeitos maiores.

Em defeito entre 3 e $10 \mathrm{~cm}$, preconiza-se o reparo laparoscópico, excetuando-se os casos de obesos ou recidiva, em que se dá preferência pela técnica aberta.

Já em defeitos maiores (10 a $15 \mathrm{~cm}$ ), o reparo aberto apresenta mais facilidade técnica. Comparando-se os posicionamentos onlay e sublay, não há diferença com relação às recidivas, ficando o cirurgião livre para escolher a técnica com a qual tenha mais experiência. Nos casos de multirrecidivadas, lesões intra-abdominais e pacientes obesos mórbidos, é prudente o uso de técnica inlay.

Para defeitos maiores que $15 \mathrm{~cm}$, indicamos o estudo da pressão intra-abdominal. Se a pressão for menor que $10 \mathrm{cmH} 2 \mathrm{O}$, tratase a $\mathrm{HI}$ com cirurgia aberta, semelhante ao proposto para defeito entre 10 e $15 \mathrm{~cm}$. Quando a pressão estiver entre 10 e $25 \mathrm{cmH} 2 \mathrm{O}$, com a intenção de aumentar o volume da cavidade abdominal, permitindo o fechamento sem tensão da parede abdominal, indica-se o pneumoperitônio progressivo préoperatório ou a injeção de toxina botulínica tipo A na parede abdominal pré-operatória (escolha conforme disponibilidade e preferência do cirurgião).

No caso de pressão maior que $25 \mathrm{cmH} 2 \mathrm{O}$, temos o diagnóstico de uma síndrome compartimental abdominal (SCA). Uma vez diagnosticada a SCA, o tratamento padrão ouro estabelecido é a descompressão cirúrgica por meio de laparotomia xifopúbica mediana, deixando em peritoniostomia, utilizando-se uma técnica de fechamento temporário (suficiente para fornecer a descompressão da fáscia abdominal).

O fechamento abdominal temporário ótimo não deve prejudicar a fáscia, a aponeurose ou a pele e deve facilitar a aproximação gradual da pele. A discussão detalhada sobre a abordagem da peritoniostomia não faz parte do escopo deste artigo.

Tal esquema de condução poderia ser adotado em qualquer centro hospitalar que cuide de tal patologia, uma vez que ele segue as recomendações vigentes em vários artigos científicos especializados no tema.

\section{REFERÊNCIAS}

1. Speranzini $M B$, Deutsch $C R$. Grandes hérnias incisionais. $A B C D$, arq bras cir dig. 2010 Oct-Dez;23(4):280-6. doi: http://dx.doi.org/10.1590/S010267202010000400015 .

2. Khaiara HS, Lall P, Hunter B, Brown JH. Repair of incisional hernias. J R Coll Surg Edinb. 2001Feb;46(1):39-43. PubMed PMID: 11242742.

3. Timmermans L, Goede B, Eker HH, van Kempen BJ, Jeekel J, Lange JF. Metaanalysis of primary mesh augmentation as prophylactic measure to prevent incisional hernia. Dig Surg. 2013;30(4-6):401-9. doi: 10.1159/000355956. PubMed PMID: 24217341.

4. Antoniou SA, Antoniou GA, Antoniou Al, Granderath FA. Past, present, and future of minimally invasive abdominal surgery. JSLS. 2015 Jul-Sept;19(3): e2015.00052. PMC4589904.

doi: 10.4293/JSLS.2015.00052. PubMed Central PMCID:

5. Hoer J, Lawong G, Klinge U, Schumpelick V. Factors influencing the development of incisional hernia: a retrospective study of 2,983 laparotomy patients over a period of 10 years. Chrirug. 2002 May;73(5):47480. doi: 10.1007/s00104-002-0425-5. PubMed PMID: 12089832.

6. Rabinerson D, Avrech $O$, Neri A, Schoenfeld A. Incisional hernias after laparoscopy. Obstet Gynecol Surv. 1997 Nov;52(11):7013. PubMed PMID: 9357043.

7. Downes, RO. Single incision laparoscopic primary and incisional ventral hernia repair as the standard of care in the ambulatory setting; Does less equal 
better outcomes; Case series and literature review. Int J Surg Case Rep. 2016; 26: 73-76. doi: 10.1016/j.ijscr.2016.07.015. PubMed PMID: 27471095.

8. Gutiérrez de la Peña C, Vargas Romero J, Diéguez Garcia JA. The value of $\mathrm{CT}$ diagnosis of hernia recurrence after prosthetic repair of ventral incisional hernias. Eur Radiol. 2001;11(7):1161-4. PubMed: 11471605.

9. Beck WC, Holzman MD, Sharp KW, Nealon WH, Dupont WD, Poulose BK. Comparative effectiveness of dynamic abdominal sonography for hernia vs computed tomography in the diagnosis of incisional hernia. J Am Coll Surg. 2013;216(3):447-453. doi: 10.1016/j.jamcollsurg.2012.11.012.

10. Chevrel JP, Rath AM. Classification of incisional hernias of the abdominal wall. Hernia. 2000; 4(1):7-11. doi:10.1007/BF01230581.

11. Silva AL. Hernias da parede abdominal. São Paulo: Atheneu; 1997. v. 1

12. Muysoms FE, Miserez M, Berrevoet F, Campanelli G, Champault GG, Chelata $E$, et al. Classification of primary and incisional abdominal wall hernias. Hernia. 2009 Aug; 13(4): 407-14. doi: 10.1007/s10029-009-0518-x.

13. Baccari P, Nifosi J, Ghirardelli L, Staudacher C. Laparoscopic incisional and ventral hernia repair without sutures: a single-center experience with 200 cases. J Laparoendosc Adv Surg Tech A. 2009 Apr; 19(2):175-9. doi: 10.1089/ lap.2008.0244. PubMed PMID: 19216699.

14. Bratzler DW, Dellinger EP, Olsen KM, Perl TM, Auwaerter PG, Bolon M K et al. Clinical practice guidelines for antimicrobial prophylaxis in surgery. Am J Health Syst Pharm. 2013 Feb;70(3):195-283. doi: 10.2146/ajhp120568. PubMed PMID: 23327981.

15. Burger JWA, Lange JF, Halm JA, Kleinrensink GJ, Jeekel H. Incisional hernia: early complication of abdominal surgery. World J Surg. 2005 Dec;29(12):160813. doi: 10.1007/s00268-005-7929-3. PubMed PMID: 16311846.

16. Shell DH 4th, De La Torre J, Andrades P, Vasconez LO. Open repair of ventral incisional hernias. Surg Clin North Am. 2008 Feb; 88(1):61-83. doi: http://dx.doi. org/10.1016/j.suc.2007.10.008.

17. Sugerman HJ, Kellum Jr. JM, Reines HD, DeMaria EJ, Newsome HH, Lowry JW. Greater risk of incisional hernia with morbidly obese than steroid-dependent patients and low recurrence with prefascial polypropylene mesh. Am J Surg. 1996 Jan;171(1):80-4. doi: 10.1016/S0002-9610(99)80078-6. PubMed PMID: 8554156.

18. Eid GM, Mattar SG, Hamad G, Cottam DR, Lord JL, Watson A, et al. Repair of ventral hernias in morbidly obese patients undergoing laparoscopic gastric bypass should not be deferred. Surg Endosc. 2004 Feb; 18(2):207-10. doi: 10.1007/s00464-003-8915-1. PubMed PMID: 14691700.

19. Ching SS, Sarela AI, Dexter SP, Hayden JD, McMahon MJ. Comparison of early outcomes for laparoscopic ventral hernia repair between nonobese and morbidly obese patient populations. Surg Endosc. 2008 Oct; 22(10):2244-50. doi: 10.1007/s00464-008-0039-1. PubMed PMID: 18622552.

20. Vilallonga R, Fort JM, Gonzalez O, Baena JA, Lecube A, Armengol M. Management of patients with hernia or incisional hernia undergoing surgery for morbid obesity. J Obes. 2011;2011:860942. doi: 10.1155/2011/860942. PubMed Central PMCID: PMC2653214.

21. Rahal F, Birolini D. Pode a h érnia inguinal transformar-se em um dilema?. Rev Assoc Méd Bras. 2001 Jan-Mar; 47(1):10. doi: http://dx.doi.org/10.1590/ S0104-42302001000100011.

22. Thompson MJ. Parastomal hernia: incidence, prevention and treatment strategies. Br J Nurs. 2008;17(2):S16, S18-20. PubMed PMID: 18418932.

23. Cuccurullo D, Piccoli M, Agresta F, Magnone S, Corcione F, Stancanelli V, Melotti G. Laparoscopic ventral incisional hernia repair: evidence-based guidelines of the first Italian Consensus Conference. Hernia. 2013 Oct;17(5):55766. doi: 10.1007/s10029-013-1055-1. PubMed PMID: 23400528.

24. Sauerland S, Walgenbach $M$, Habermalz B, Seiler CM, Miserez $M$. Laparoscopic versus open surgical techniques for ventral or incisional hernia repair. Cochrane Database Syst Rev. 2011 Mar 16; (3):CD007781. doi: $10.1002 / 14651858$.

25. Berger D, Bientzle M, Müller A. Postoperative complications after laparoscopic incisional hernia repair. Incidence and treatment. Surg Endosc. 2002;16(12):1720-3. doi: 10.1007/s00464-002-9036-y. PubMEd PMID: 12209325

26. Mudge $M$, Hughes LE. Incisional hernia: a 10year prospective study of incidence and attitudes. Br J Surg. 1985 Jan;72(1):701. PubMed PMID: 3155634.

27. Park AE, Roth S, Kavic M. Abdominal wall hernia. Curr Probl Surg. 2006 May; 43(5):326-75. PubMed: 16679124. doi: 10.1067/j.cpsurg.2006.02.004.

28. Schumpelick V, Junge K, Rosch R, Klinge U, Stumpf M. Retromuscular mesh repair for ventral incision hernia in Germany. Chirurg. 2002 Sep;73(9):888-94. doi:10.1007/s00104-002-0535-0. PubMed PMID: 12297953.

29. Helgstrand F, Rosenberg J, Kehlet H, Jorgensen LN, Bisgaard T. Nationwide prospective study of outcomes after elective incisional hernia repair. J Am Coll Surg. 2013 Feb; 216(2):217-28. doi: 10.1016/j.jamcollsurg.2012.10.013. PubMed PMID: 23219350.

30. Mahmoud Uslu HY, Erkek AB, Cakmak A, Sozener U, Soylu L, Turkcapar AG, Kuterdem E. Incisional hernia treatment with polypropilene graft: result of 10 years. Hernia. 2006 Oct;10(5):380-4. doi: 10.1007/s10029-006-0107-1. PubMed PMID: 16841147.

\section{Como citar este artigo/How to cite this article:}

Markovic A, Barreira MA, Goes ACAM. Hérnia Incisional: proposta de um fluxograma que oriente o tratamento. J Health Biol Sci. 2016 Out-Dez; 4(4):257264 . 